\title{
Caractérisations Granulométrique et Paléo- Environnementale des Sédiments d'un Bassin Versant à Forte Dynamique Structurale dans un Contexte de Ravinement: Bassin Versant de Ourossogui (Nord du Sénégal)
}

\author{
Dr. Seydou Alassane Sow,
}

Laboratoire Leïdi « Dynamiques des territoires et développement », Section de Géographie, Ecosystémes et Environnement, Université Gaston Berger de

Saint-Louis, Sénégal

Dr. Amadou Abou Sy,

Université Cheikh Anta DIOP, Faculté LSH, Département de Géographie,

Dakar, Sénégal

Prof. Boubou Aldiouma Sy,

Laboratoire Leïdi «Dynamiques des territoires et développement », Section de Géographie, Ecosystémes et Environnement, Université Gaston Berger de

Saint-Louis, Sénégal

URL:http://dx.doi.org/10.19044/esj.2019.v15n32p86

Résumé

L'intérêt de cette caractérisation sédimentaire par granulométrie, est de faire une reconstitution paléo-environnementale des sédiments du bassin versant de Ourossogui. Ainsi, la méthodologie est basée sur des analyses granulométriques au laboratoire. Le traitement des données s'est fait grâce à des indices granulométriques et des diagrammes de K-Sk de Friedman et de Mz-Sk de Moiola et Weiser. Les résultats montrent que les faciès lithologiques superficiels observés dans le bassin versant de Ourossogui sont en majorité composés de particules sableuses. Quant au diagramme de K-Sk de Friedman, il renseigne que les sédiments sont apportés par les eaux courantes, alors que celui de Mz-Sk de Moiola et Weiser a montré que les sédiments sont intégralement d'origine continental, avec cependant une légère présence de sédiments d'origine marine. Ils correspondent à des apparitions de faciès de calcaires phosphatées au niveau des éboulis situés sur les lits des ravins du bassin versant. Ce contexte paléo-environnemental du bassin versant est à l'origine de sa forte dynamique structurale, marquée par une réactivation de la morphodynamique hydrique. 
Mots clés: Paléo-lithologie, Dynamique structurale, Bassin versant, Ourossogui, Vallée du Sénégal

\title{
Granulometric and Paleo-Environmental Characterizations of Sediments in a Watershed Area with Strong Structural Dynamics in a Gully Context: Ourossogui Watershed (Northern Senegal)
}

\author{
Dr. Seydou Alassane Sow, \\ Leidi Laboratory "Dynamics of territories and development", Section of \\ Geography, Ecosystems and Environment, Gaston Berger University of \\ Saint-Louis, Senegal \\ Dr. Amadou Abou Sy, \\ Cheikh Anta DIOP University, LSH Faculty, Department of Geography, \\ Dakar, Senegal \\ Prof. Boubou Aldiouma Sy, \\ Leïdi Laboratory "Dynamics of territories and development", Section of \\ Geography, Ecosystems and Environment, Gaston Berger University of \\ Saint-Louis, Senegal
}

\begin{abstract}
The interest of this sedimentary characterization by granulometry, is try to do a paleo-environmental reconstitution of the sediments of the watershed of Ourossogui. For this reason, the methodology is based on granulometric analyzes in the laboratory. Data processing was done using grain size indices and Friedman K-Sk and Moiola and Weiser Md-Sk diagrams. The results show that the superficial lithological facies observed in the Ourossogui watershed are mostly composed of sandy particles. As for the Friedman K-Sk diagram, it indicates that the sediments are brought by running waters, while that of Mz-Sk of Moiola and Weiser showed that the sediments are entirely of continental origin, with however a slight presence sediments of marine origin. They correspond to occurrences of phosphated limestone facies in the scree on the beds of the watershed ravines. This paleo-environmental context of the watershed is at the origin of its strong structural dynamics, marked by a reactivation of the water morphodynamics.
\end{abstract}


Keywords: Paleo-lithology, Structural dynamics, Watershed, Ourossogui, Senegal valley

\section{Introduction}

Le bassin versant de Ourossogui est situé au cœur de la moyenne vallée du fleuve Sénégal. Cette vallée garde une structure géomorphologique particulière qui est largement dépendante du micro-relief. Les matériaux ont été mis en place en différentes étapes : entre le Secondaire et le Quaternaire. Cette dernière ère a connu des modifications climatiques ayant influencé l'évolution du système morphogénique de la vallée. La connaissance des changements du climat au cours du Quaternaire en Afrique occidentale a ainsi intéressé de nombreux chercheurs (Tricart, 1956 ; Michel, 1973 ; Dubois, 1967 ; Giresse, 2000, etc.).

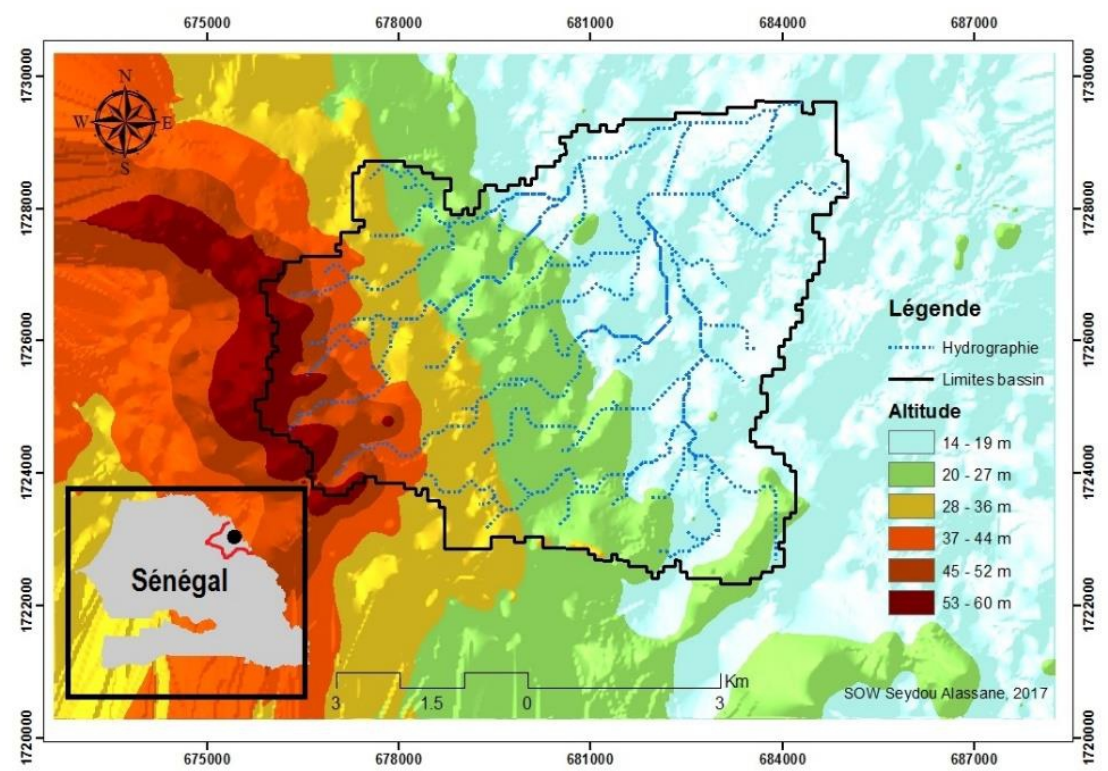

Figure 1 : Carte de localisation du bassin versant de Ourossogui

Située sur le Djédjégol $^{3}$, la ville de Ourossogui est bâtie au pied d'une chaine de petites collines, dont les sommets n'excédant pas plus de $60 \mathrm{~m}$ IGN. Le relief demeure relativement plat. Cependant, cette platitude est rompue par la présence de petites vallées qui ceinturent la ville dans ses parties Sud-est et Nord-ouest. Le réseau hydrographique est constitué d'axes hydrauliques issus de la chaine de collines située à l'Ouest, drainant souvent des quantités

${ }^{3}$ Zone de transition entre le Walo et le Diéry. Le Walo correspond à la zone des sols argileux et est à proximité du fleuve Sénégal. Le Diéry correspond à la zone des sols arides et sableux de la vallée du Sénégal. 
énormes d'eau de pluies. C'est un réseau à écoulement temporaire ; il est actif en hivernage, à la suite d'une impulsion pluviométrique. Les eaux d'écoulement sont drainées vers les secteurs dépressionnaires situés à l'intérieure de la ville.

A Ourossogui, les faciès des dépôts de couverture sont essentiellement constitués d'une superposition de grés, de sables et d'argiles. Le processus de mise en place des matériaux dans les paysages géographiques ouest-africains en général et dans la moyenne vallée du Sénégal en particulier s'est fait autour de vieilles plates-formes surplombant des bassins structuraux. Leur évolution diagénétique a donné des faciès essentiellement gréseux (dépôts de couverture), à partir desquels se sont dégagées les différentes unités géomorphologiques du Quaternaire comme les glacis du Ferlo, les buttes de Garly, les collines de Ourossogui ou des inselbergs isolés quelques part dans la moyenne vallée (Sow, 2018). L'alternance des épisodes climatiques sèche et humide a dans l'ensemble abouti à l'élaboration de matériaux meubles, qui constituent les terrains où s'exerce une intense activité morphodynamique. A cet effet, le bassin versant de Ourossogui est soumis à une forte dynamique structurale, se manifestant par des phénomènes de ravinement. Le ravinement est un processus d'écoulement des eaux de pluies dans un réseau hydrographique, organisé en ravines ou de ravins (Cosandey et Robinson, 2000). Les ravinements provoquent en effet la destruction des ouvrages de franchissement et des axes routiers, isolant ainsi par inondations certaines localités par rapport à d'autres. Ces phénomènes sont très fréquents entre la localité de Ourossogui et son hinterland. Ils entrainent aussi une érosion de berges de ravins à travers l'extension progressive de leurs têtes et des pertes en terres. Les études issues des savanes ouest-africaines (Balzerek, 2003) mettent en évidence l'intensification de cette forme de morphodynamique et de ses conséquence hydrogéomorphologiques et socioéconomiques. Les ravinements deviennent de plus en plus importants sur des espaces dénudés, avec comme corollaire l'ensablement des mares, la recharge de la nappe dans des milieux endoréiques et une érosion des versants (Bouzou Moussa et al., 2009) pouvant entrainer une redistribution granulométrique au niveau des cours d'eau à écoulement pérenne ou temporaire à l'instar des ravins de la moyenne vallée du Sénégal.

L'objectif de cet article est de faire une caractérisation granulométrique du bassin versant de Ourossogui, dont la dynamique structurale actuelle se traduit par une réactivation des processus érosifs. L'intérêt de cette caractérisation est d'établir une reconstitution paléoenvironnementale (grâce aux indices granulométriques) des sédiments de la zone d'étude. Les travaux de Friedman (1962), de Moiola et Weiser (1968), de Folk et Ward (1957), de Sakho (2011), de Tossou (2019), et de Sow (2019) ont tous développé sur ce lien intrinsèque qui existe entre caractérisation 
sédimentaire par granulométrie et paléo-environnement sédimentaire d'un milieu. Un protocole méthodologique a été adopté.

\section{Matériels et méthodes}

\subsection{Protocole d'échantillonnage des sédiments}

Les prélèvements sont intégralement effectués en surface. En pratique, le protocole consiste à faire des extractions d'échantillons suivant une profondeur maximale de $20 \mathrm{~cm}$ et codés sur la base des unités sur lesquelles ont eu lieu le prélèvement $(\mathrm{ZC}=$ Zone des collines, $\mathrm{RV}=$ Ravins, $\mathrm{ZE}=\mathrm{Z}$ Zne d'épandage, $\mathrm{CV}=$ Centre-ville). L'échantillonnage bien qu'étant aléatoire respecte une toposéquence orientée en fonction des transects. Au niveau de la zone collinaire et dans le centre-ville, les transects suivent une direction Nordsud. Cependant, au niveau des deux principaux ravins, ces transects prennent une direction similaire à celle des accumulations de flux dans le bassin versant (Ouest-est). Chaque transect comporte 10 points de prélèvement, soit un total de 40 échantillons, en raison de 10 échantillons par transect. L'objectif de cet échantillonnage est de faire un traitement granulométrique des sédiments. L'ensemble des points échantillonnés durant les deux campagnes de récolte de sédiments sont géoréférencés à l'aide d'un GPS.

\subsection{Analyses au laboratoire}

Au laboratoire, une colonne de 8 tamis superposés selon un ordre de grandeur pour filtrer respectivement. Au laboratoire, une colonne de 8 tamis superposés selon un ordre de mailles décroissant du haut en bas pour filtrer respectivement les cailloux, le sable très grossier, le sable grossier, le sable moyen, le sable fin, et le sable très fin, mais aussi les limons et les argiles. Le travail de tamisage et de filtrage s'est effectué grâce à un oscillateur granulométrique de type FRITSCH. Après chaque séance d'agitation, la masse contenue dans chaque colonne de tamis est pesée à la balance électrique. Les résultats obtenus sur la granulométrie sont traités par des analyses statistiques. Les sédiments ont été caractérisés à travers la moyenne granulométrique $(\mathrm{Mz})$, le Sorting index ou indice de tri ou de classement $\left(\mathrm{O}_{\mathrm{i}}\right)$, le Skewness ou indice d'asymétrie (Ski) et le Kurtosis ou l'indice d'acuité (KG). Ces paramètres statistiques ont été obtenus grâce au logiciel Origine 6. Le même outil a été utilisé pour générer les courbes cumulatives de la granulométrie. La dispersion des points permet de mettre en relation la granulométrie et l'environnement de dépôt des sédiments.

Les méthodes utilisées ont permis d'obtenir des résultats qui ont permis de faire le lien entre la lithologie et la dynamique structurale du bassin versant de Ourossogui. 


\section{Résultats}

Les résultats obtenus permettent de faire le point sur les caractéristiques granulométriques des sédiments et leur paléo-environnement.

\subsection{Caractéristiques granulométriques des sédiments du bassin versant de Ourossogui}

Les faciès lithologiques du bassin versant de Ourossogui sont en majorité composés de sables. Les analyses sont mises en évidence par des indices granulométriques.

- La moyenne granulométrique (Mz) : elle représente la distribution granulométrique moyenne des sédiments et permet l'individualisation d'un certain nombre de faciès. Il renseigne sur l'énergie cinétique moyenne lors du dépôt des sédiments.

Selon la terminologie de Folk et Ward, sa formule est : $M_{z}=\frac{\emptyset_{16}+\emptyset_{50}+\emptyset_{84}}{3}$

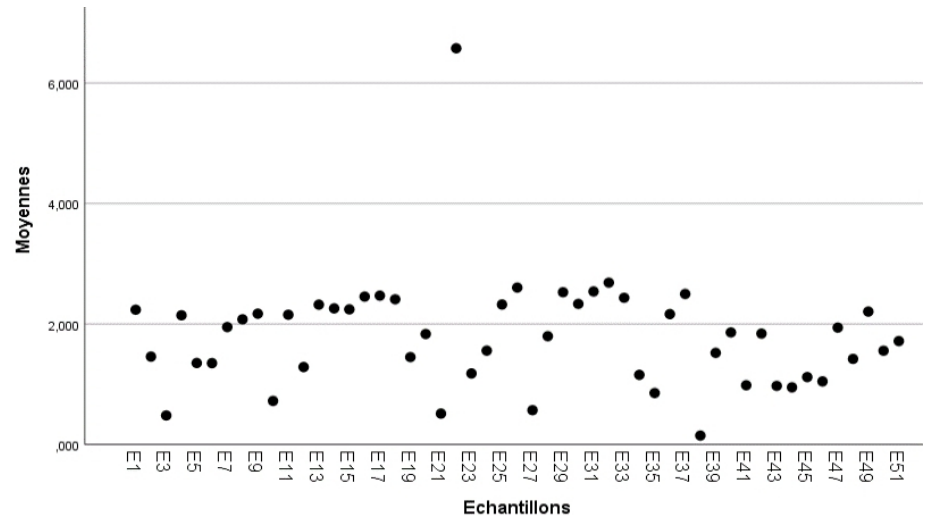

Figure 2 : Distribution de la moyenne granulométrique des sédiments prélevés

L'analyse de la distribution de la moyenne granulométrique des faciès sédimentaire du bassin versant de Ourossogui révèle que la plupart des sédiments sont situés entre la fourchette 1 et 2,9; confirmant ainsi la prédominance de la fraction sableuse. Sur la colonne de tamis utilisée, cette fraction va de $0,1 \mu \mathrm{m}$ et $3,32 \mu \mathrm{m}$ : il s'agit des sables fins, des sables moyens, et des sables grossiers et graviers.

- Le Sorting Index ou indice de tri ou de classement $\left(\sigma_{i}\right)$ : il renseigne sur l'hydrodynamisme lors du dépôt du sédiment. Il indique la qualité du classement ; plus il est faible, plus le sédiment est bien classé. Selon Folk et Ward, sa formule est :

$$
\sigma_{i}=\frac{\left(\emptyset_{84}-\emptyset_{16}\right)}{4}+\frac{\left(\emptyset_{95}-\emptyset_{5}\right)}{6,6}
$$

La figure 4 qui suit montre le classement des échantillons de sédiments dans le bassin versant de Ourssogui. 


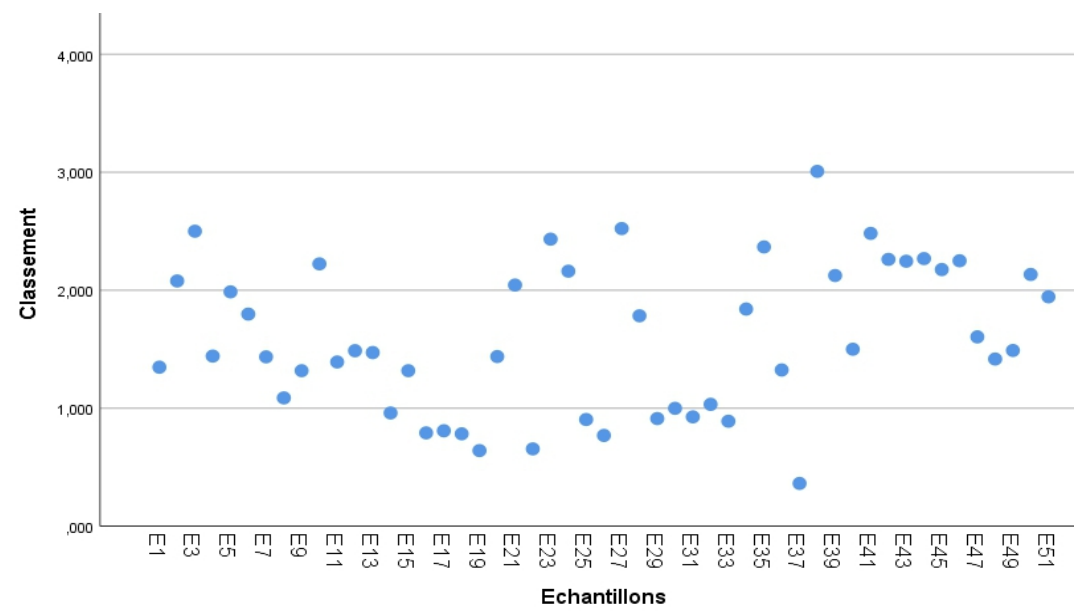

Figure 3 : Distribution de l'indice de classement des sédiments du bassin versant de Ourossogui

L'analyse de la figure 4 montre que la plus part des sédiments sont situés entre les intervalles 0,5 et 2,5. Ce qui signifie que les sédiments du bassin versant sont en partie moyennement classés mais aussi regorge une bonne partie de particules médiocrement classées. De tels indices de classement sont caractéristiques des environnements à influences d'érosion hydrique.

- Le Skewness ou indice d'asymétrie ( $\left.\boldsymbol{S} \boldsymbol{k}_{\boldsymbol{i}}\right)$ : Ce paramètre informe sur l'enrichissement en particules grossières (asymétrie négative), l'enrichissement en particules fines mal triées (asymétrie positive), ou s'il y a symétrie (Skewness compris entre -0.10 et 0.10 ). Sa formule est selon Folk et Ward :

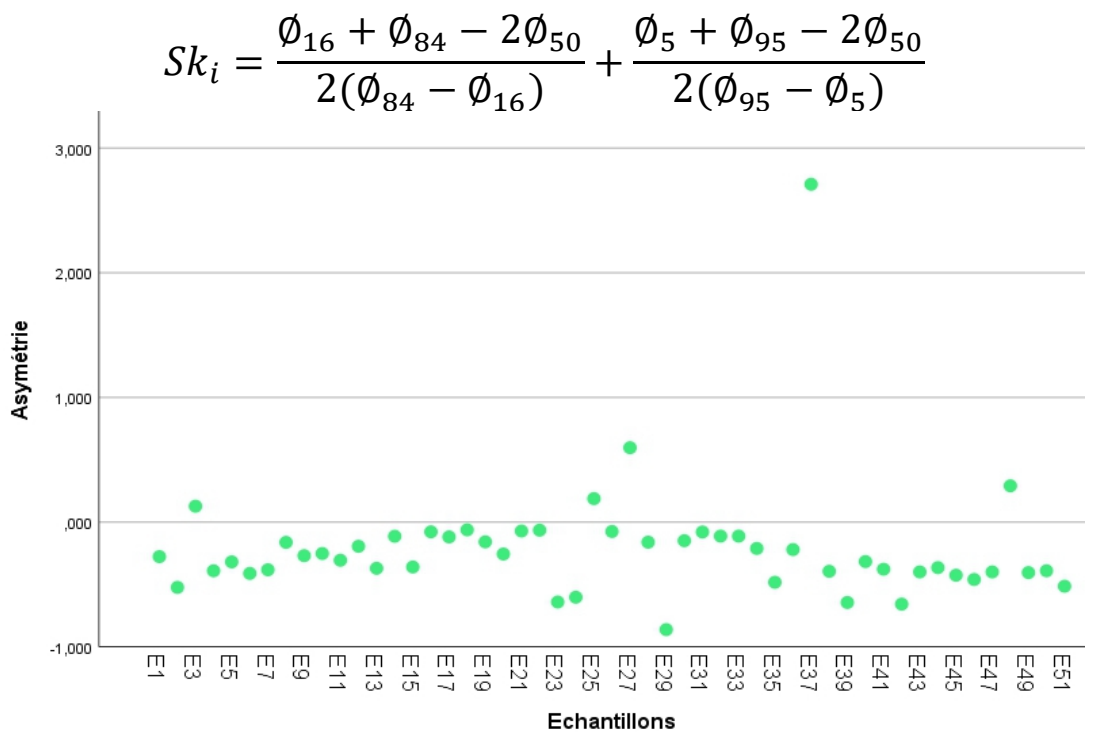

Figure 4 : Distribution de l'indice d'asymétrie des sédiments prélevés 
Les sédiments du bassin versant de Ourossogui sont marqués surtout par une asymétrie négative (enrichissement en particules grossières), suivant la terminologie de Folk et Ward (1957). Ce qui témoigne de l'influence d'une seule source d'apport sédimentaire dans ce milieu : les eaux de ruissellement et de ravinement.

- Le kurtosis ou l'indice d'acuité $\left(K_{G}\right)$ : c'est l'indice d'acuité du mode, il mesure l'angulosité de la courbe des fréquences. Si le mode est concentré dans des classes granulométriques restreinte, on a une distribution leptocurtique, s'il est dispersé on a une distribution platicurtique. D'autres types de distribution peuvent néanmoins exister aussi. Sa Formule est :

$$
K G=\frac{\varnothing 95-\varnothing 5}{2,44(\varnothing 75-\varnothing 25}
$$

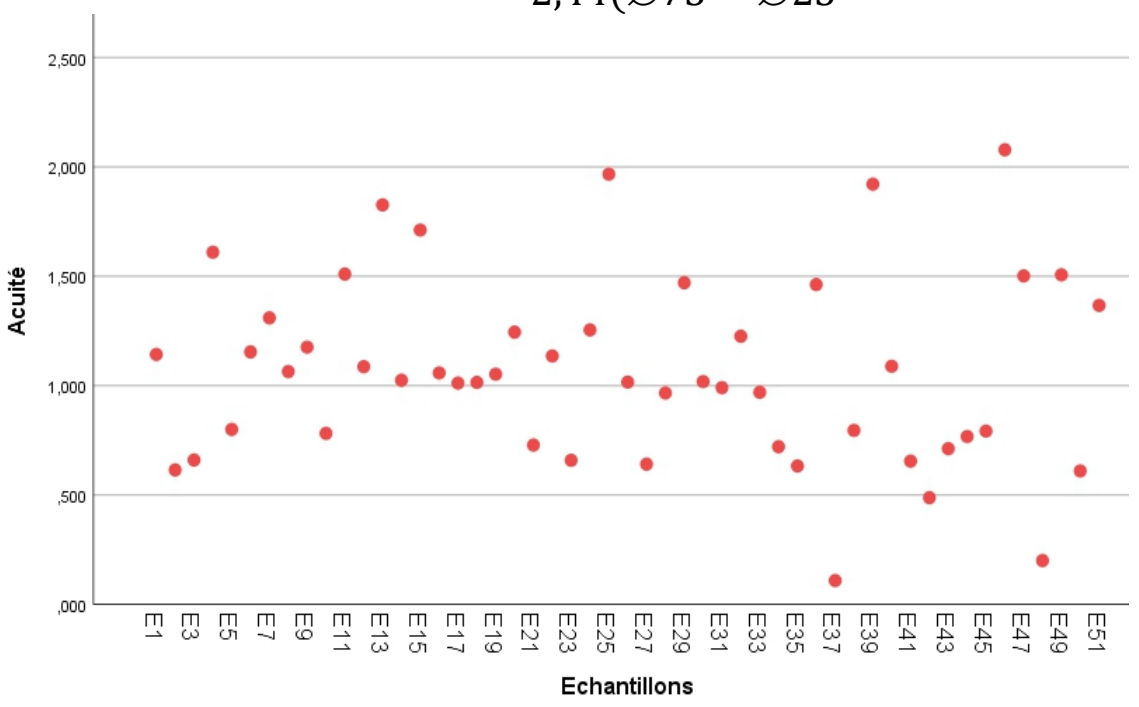

Figure 5 : Distribution de l'indice d'acuité des sédiments prélevés

L'indice d'acuité des sédiments du bassin versant de Ourossogui évolue de la classe des courbes platycurtique à leptocurtique en passant par mésocurtique. Cette répartition témoigne des variations influençant le régime sédimentaire du milieu. Les sédiments sont certes déposés en partie par des eaux courantes, mais parfois aussi par l'agent éolien.

La caractérisation granulométrique s'est aussi faite par les percentiles que la figure 6 qui suit essaye de mettre en évidence. 


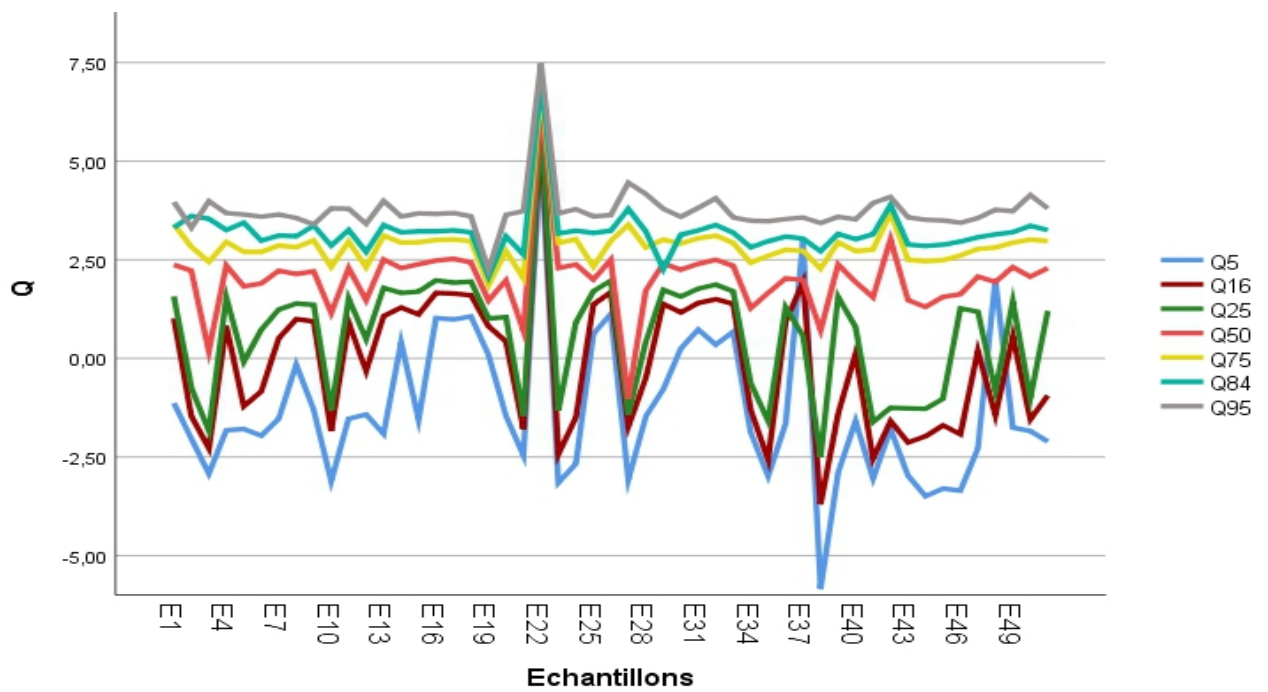

Figure 6 : Distribution des percentiles granulométriques des sédiments du bassin versant de Ourossogui

Cette figure 7 qui précède permet de mettre en évidence une première série de paramètres, desquels découlent tous les autres. Ce sont les percentiles $Q x$, points correspondants à des mailles de tamis hypothétiques sur lesquelles $x \%$ en poids du sédiment est de dimension plus grande (ou plus petite). Les valeurs d'abscisse utilisées sont : $\mathrm{Q}_{5} ; \mathrm{Q}_{16} ; \mathrm{Q}_{25} ; \mathrm{Q}_{50} ; \mathrm{Q}_{75} ; \mathrm{Q}_{84}$ et $\mathrm{Q}_{95}$. Elles permettent d'explorer la série et de ressortir toutes les formes caractéristiques des sédiments suivant différentes sections. Ainsi, l'analyse de la figure 7 permet de constater que toutes les valeurs des percentiles suivent une allure analogue et varient entre $-5,84$ et 7,477. La médiane ou $Q_{50}$ est l'indice de position le plus fréquemment employé car, il donne en intégrant toutes les classes granulométriques une estimation de la grossièreté moyenne du sédiment. La faiblesse du $\mathrm{Q}_{50}$ à l'échelle de tous les échantillons est un paramètre indicateur de la prédominance de la fraction sableuse dans le bassin versant.

L'analyse granulométrique des sédiments échantillonnés au niveau du bassin versant de Ourossogui est suivie d'une exploration de leur paléoenvironnement afin de revisiter leur milieu et modes de dépôt.

\subsection{Paléo-environnement des sédiments du bassin versant de Ourossogui : milieu et mode de dépôt}

L'alternance des faciés à texture différente dans le bassin versant de Ourossogui est mise en évidence par la figure 8 et qui permet d'observer la chronostratigraphie des sédiments sur une partie du bassin versant de Ourossogui. 


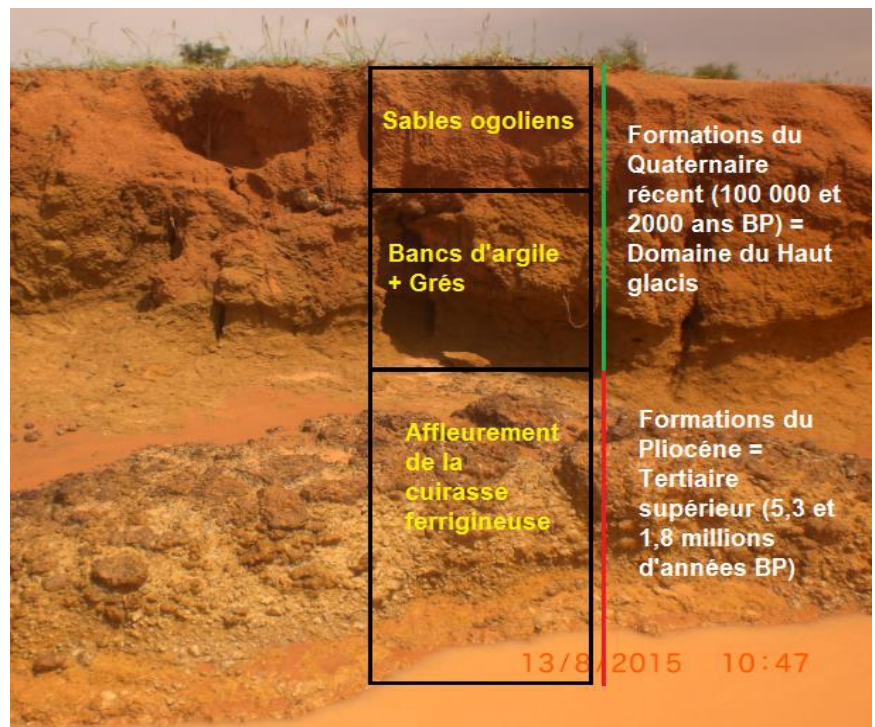

Figure 7 : Chronostratigraphie des faciés sur le ravin du Sud-est du bassin versant de Ourossogui

La figure 8 établit une chronostratigraphie des faciès du ravin qui jouxte la partie Sud-est du bassin versant de Ourossogui. Elle reflète la nature tendre des faciès qui affleurent: sables et argiles gréseux du Quaternaire récent.

Les deux premières couches sont formées de sables et d'argiles entremêlés de grés. La couche sableuse est constituée d'un mélange de diverses espèces minérales comme l'aluminium et le fer. Il s'agit des marques de l'Ogolien (22000 ans à 12000 ans BP). Ce sol a la propriété de devenir hydro-gonflante quand il est imbibé d'eau. Ses particules forment des agrégats qui délimitent une zone poreuse où on observe de grandes cavités dont les caractéristiques sont fonction de la répartition de la matière rocheuse (texture) et de l'agencement des matériaux (structure). Sa capacité d'infiltration est relativement importante du fait de sa texture grossière et de sa structure fragmentaire. Quant à l'argile, sa texture compacte montre des particules très fines, de taille différente imbriquées les unes dans les autres sous la forme de lame : cette roche a la propriété de devenir hydrogonflante quand elle est imbibée d'eau. Sur la partie inférieure de l'image, affleurent des symboles de la cuirasse du Pliocène. Cette cuirasse est à l'origine ferrugineuse, et constituait un revêtement sommital dur dont, les processus diagénétiques ont été influencés par l'oxydation du fer dans une alternance d'épisodes morphoclimatiquement aride et humide. Son démantèlement a entrainé la mise en place des éboulis dans différents endroits le long des ravins comme le témoigne la photo 3 qui suit. 


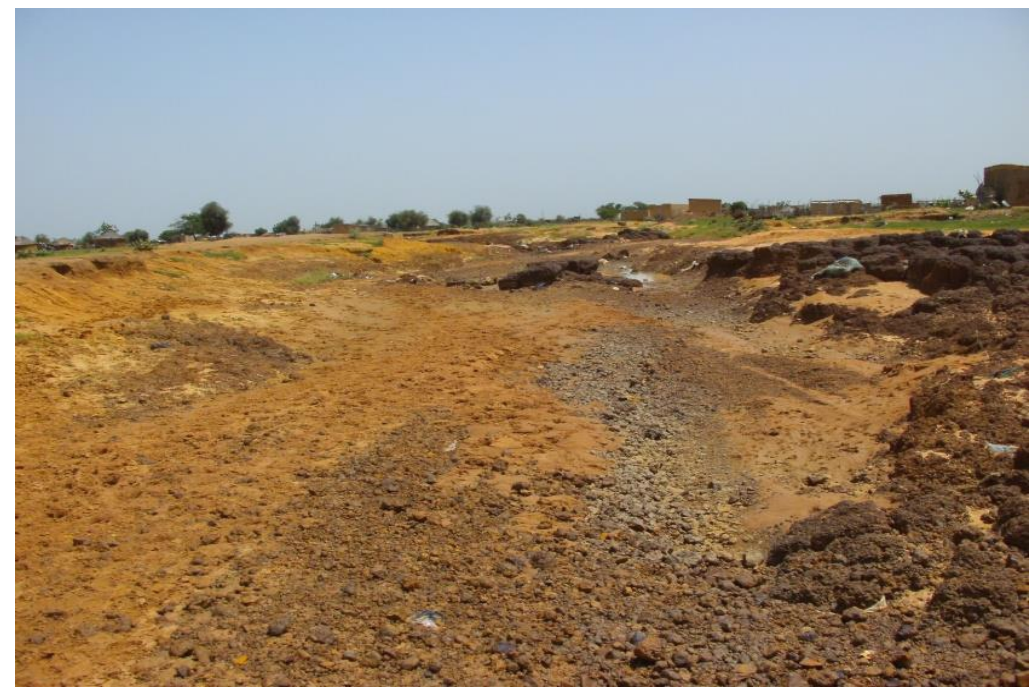

Photo 1 : Eboulis sur une partie du ravin du Nord-est

La superposition des couches de cette série sédimentaire d'origine continentale, permet d'établir une échelle de résistance pour apprécier la résistance de ces affleurements au regard de la dynamique structurale du bassin versant. La résistance est liée à la cohésion, à la solubilité et à l'homogénéité de la roche. La cohésion renvoie à la plus ou moins grande résistance aux tensions susceptibles de rompre la roche ou encore la plus ou moins grande solidité des liens (ciments) autour desquels s'agglomèrent les minéraux constituant la roche.

L'analyse des courbes granulométriques des sédiments permet de mieux comprendre les caractéristiques des sédiments des couches de cette série sédimentaire.
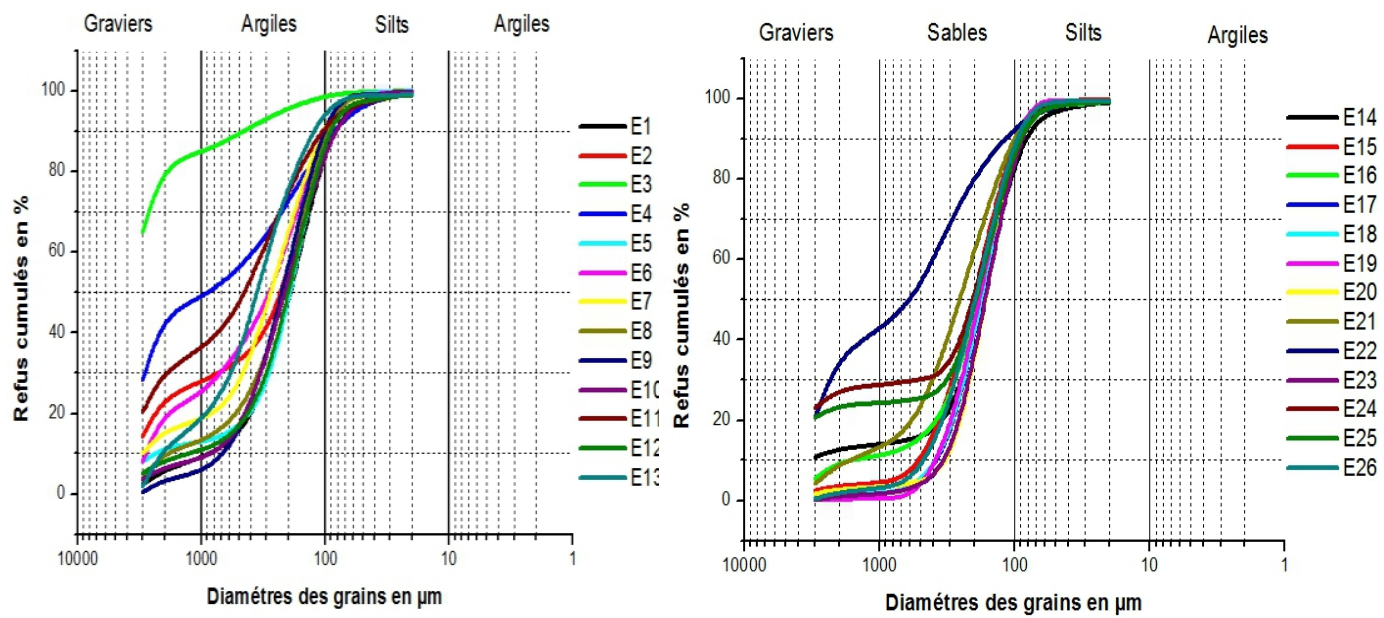

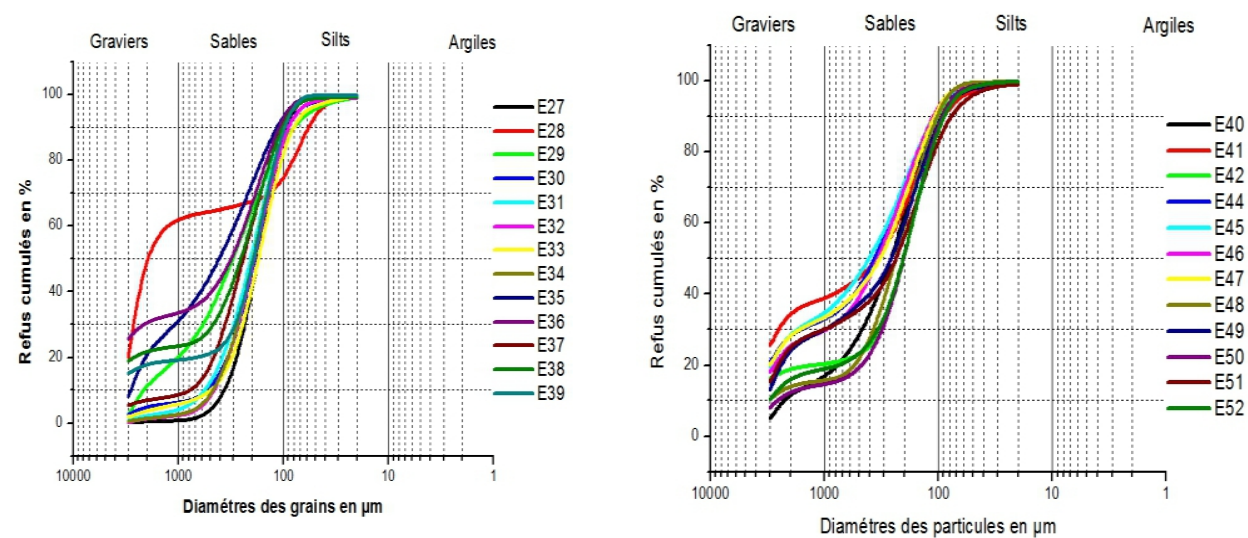

Figure 8 : Courbes granulométriques des sédiments prélevés dans le bassin versant de Ourossogui

Les courbes semi-logarithmiques des sédiments de la zone d'étude ont une allure quasi-hyperbolique. Elles sont caractéristiques des zones continentales calmes où toute particule apportée par l'eau est définitivement bloquée à la suite d'un ralentissement de la vitesse du flux hydrique en transit. Il s'agit ici des faciès de décantation dans des unités morphologiques fonctionnant comme des zones de collecte d'eau de ruissellement (ravins), et par conséquent de piège de sédiments. La fin de la distribution vers les fines se traduit par une limite très tranchée aux environs de $0,02 \mu \mathrm{m}$ : correspondant ici à des argiles.

Les diagrammes K-Sk de Friedman (figure 9), et ceux Mz-Sk de Moiola et Weiser (figure 10), ont été utilisés pour déterminer l'origine des sédiments du bassin versant de Ourossogui.

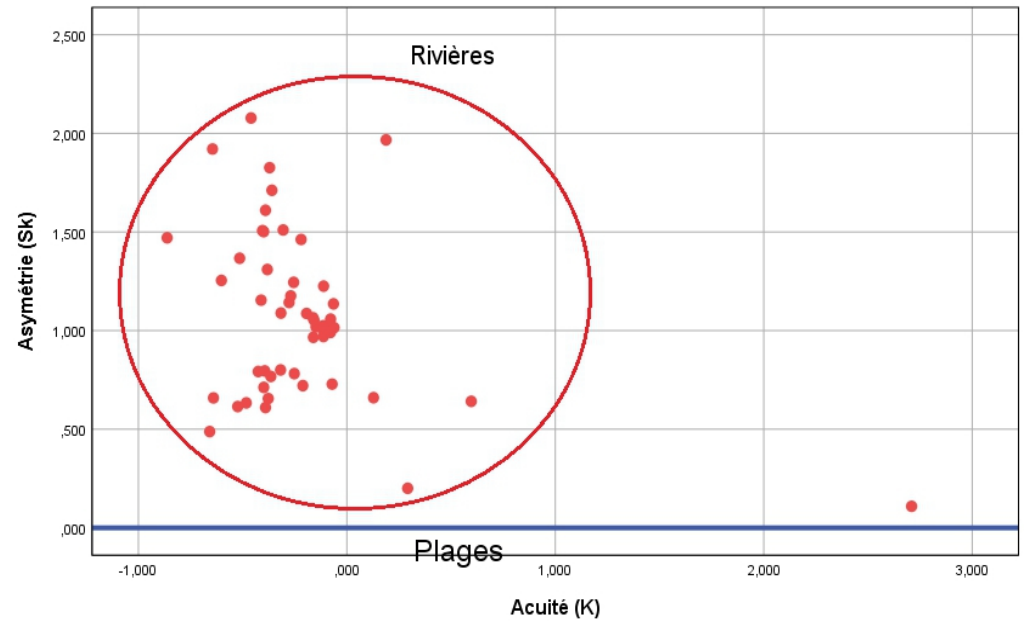

Figure 9 : Diagramme K-Sk de Friedman des sédiments du bassin versant de Ourossogui 
L’analyse du diagramme K-Sk de Friedman, montre que les sédiments du bassin versant de Ourossogui sont intégralement apportés par les eaux courantes. Quant aux diagrammes Mz-Sk de Moiola et Weiser, ils confirment les apports en provenance des environnements continentaux, avec cependant quelques sédiments issus du milieu marin.

L'apport par les eaux courantes des sédiments dans le bassin versant de Ourossogui, se justifie par le fait que le débit de pointe des eaux peut théoriquement augmenter, soit par une augmentation du coefficient de ruissellement, ou bien d'une modification de la configuration des précipitations en son sein. Dans ce dernier cas, deux hypothèses peuvent être avancées. Soit il y a une augmentation de la durée de la pluie, ce qui peut entraîner une amplification des flux d'eau ruisselants en continu plus qu'avant suite à la longue distance de connectivité entre la ligne de partage d'eau et les talwegs des axes hydrauliques (ravins), soit il y a une augmentation de l'intensité des pluies de pointe. L'hypothèse d'une production conjointe de ces deux cas de figures n'est pas à exclure. L'accroissement des coefficients de ruissellement engendre une alternance des processus d'érosion et de sédimentation, justifiant ainsi le transport par les eaux courantes des sédiments du bassin versant.

L’organisation des écoulements dans le bassin de Ourossogui varie en fonction des sections du bassin. Dans les parties hautes du bassin versant, les écoulements engendrent des ravinements dont les évolutions sont rapides et les formes variables en fonction des caractéristiques de pente du bassin versant. Le réseau de ravines s'accompagne d'une complexification, qui se traduit par une multiplication de leurs points de jonction. Ceci a pour conséquence un morcellement des versants en interfluves plus ou moins vastes. A mi-versant, correspondant à la partie aval de la zone collinaire et à la tranchée urbaine, une galerie d'obstacles freine les écoulements. La conséquence est un effet de refoulement des ondes de crue, inondant ainsi certains sites situés à proximité. A l'aval de cette zone, la morphodynamique peut reprendre sous l'effet combiné de la torrentialité de certains axes ne rencontrant pas d'obstacles sur leur trajet et/ou des échappées de crues, conditionnées par certaines ouvertures de ponts ou d'obstructions latérales sur la RN2. A l'exutoire du bassin versant, se trouve les mares et les cuvettes argileuses du Walo. Ce sont elles qui accueillent les volumes hydrosédimentaires transférés depuis l'amont du bassin et le plateau polygénique du Ferlo. Par le même processus que celui observé à mi- versant, des zones d'épandages de taille croissante s'établissent dans ces mares. Il se produit progressivement dans certains endroits un comblement par des matériaux sédimentaires de granulométries différentes mais à dominance sableuse. La photo 4 qui suit montre des sédiments de sables charriés par des eaux de ruissellement transitant par les ravins. 


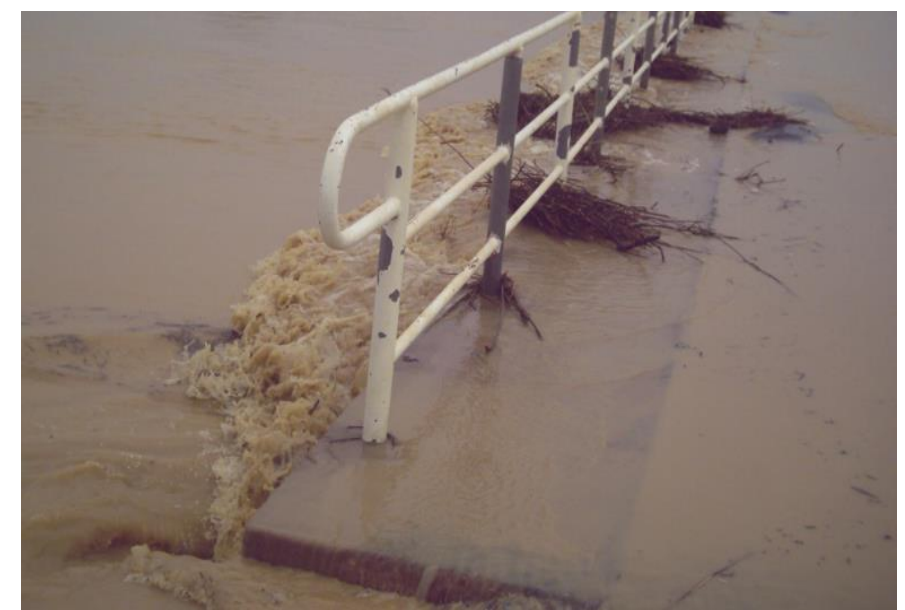

Photo 2 : Acheminement des sédiments charriés par des eaux courantes en provenance du plateau polygénique du Ferlo

La figure 11 qui suit montre l'origine des sédiments.

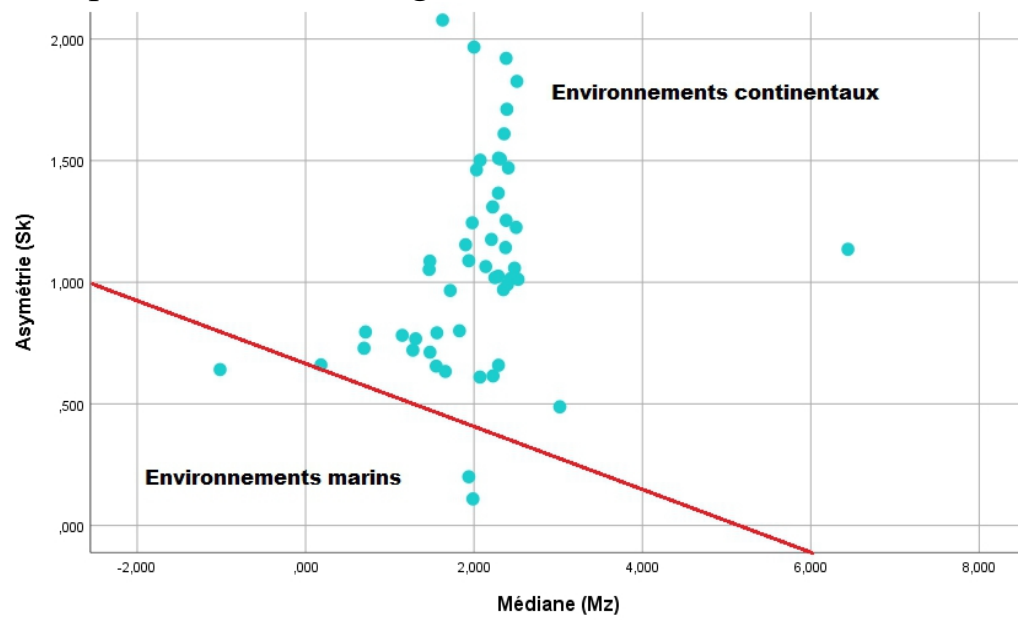

Figure 10 : Diagramme Mz-Sk de Moiola et Weiser des sédiments du bassin versant de Ourossogui

Le diagramme de Mz-Sk de Moiola et Weiser a été utilisé pour déterminer l'origine des sédiments. Son analyse montre que la quasi-totalité des sédiments sont issus des environnements continentaux. Cependant, seulement trois échantillons d'origine marine sont identifiés. Deux hypothèses peuvent être formulées quant à présence de ces sédiments à genèse marine au cœur d'un milieu continental :

- Ils pourraient être entrainés par un transport hydro-sédimentaire induit par les eaux de ruissellement. En effet, la situation géographique du bassin versant de Ourossogui dans la zone du Diéjégol lui confère la position de couloir de passage des flux hydro-sédimentaires sous 
l'effet des ravinements en provenance d'une part du Ferlo ${ }^{4}$, lui-même connecté à d'anciennes zones estuariennes ou de golfs nouacktchotiens ${ }^{5}$, qui lui sont à leur tour connectés par un système hydrographique souvent fonctionnel à la suite de sollicitation pluviométrique.

- Ils pourraient aussi correspondre à des résidus de calcairo- gréseux ou de calcaires phosphatés identifiés souvent dans les lits des ravins. Le calcaire étant un faciès sédimentaire se formant dans un environnement marin. Par ailleurs, la photo 8 avait signalé que la cuirasse ferrugineuse du Pliocène à l'origine avait évolué au gré des circonstances morpho-climatiques (aride et humide). Elle est a donc été témoin de plusieurs fluctuations climato-environnementales ${ }^{6}$ ayant abouti à des formations de faciès d'âge et d'origine différentes dans toute la vallée du Sénégal. Par ailleurs, des sondages de prospection avaient signalé la présence de phosphates dans différentes parties de la moyenne vallée du Sénégal (F. Jacquet, 1936 ; BRGM, 1965 et 1984). A proximité (Ndendory, moins de $50 \mathrm{~km}$ de Ourossogui), des gisements de phosphates sont déjà en cours d'exploitation.

\section{Discussions}

La caractérisation sédimentaire par granulométrie du bassin versant de Ourossogui a montré une prédominance de la fraction sableuse. La combinaison des indices granulométriques revêt une grande signification paléo-environnementale, et permet de distinguer des sédiments en provenance de sources différentes (Friedman, 1962 ; Moiola et Weiser, 1968). Selon Moiola et Weiser (1968), la combinaison entre le diamètre moyen (Mz) et l'asymétrie (Sk) permet de faire la distinction entre les sédiments des environnements marins et continentaux. Alors que le rapport entre $\mathrm{Mz}$ et le classement $\left(\sigma_{\mathrm{i}}\right)$ est plus significatif pour la discrimination de l'enrichissement par mode de transport du sable apportés par les plages ou des eaux courantes. A cet effet, ces indices granulométriques ont montré les ressemblances et différences qui peuvent exister dans les caractéristiques sédimentologiques des différents échantillons.

L'étude paléo-environnementale des sédiments a révélé des origines continentales détritiques à dominance sables moyens et fins qui jonchent les lits de ravins. Les sédiments sont intégralement apportés par les eaux courantes. Ces eaux sont le résultat d'une collection de flux en partance du plateau polygénique du Ferlo et transitant vers le Diéjogol, site transitoire

\footnotetext{
${ }^{4}$ Ancienne vallée fonctionnelle devenue fossile.

${ }^{5}$ Anciens golfs marins formés durant un épisode morphoclimatique située entre 8000 ans et 6500 ans BP et marquée par une transgression de la mer à l'intérieur du continent.

${ }^{6} \mathrm{Du}$ Tertiaire supérieur au Quaternaire récent
} 
entre le Walo argileux et le Diéry sableux. Seulement quelques échantillons signalent une genèse marine en plein cœur d'un milieu continental. Ceci est consécutif à la géologie du milieu et d'autre part à son évolution géomorphologique. La présence des éboulis sur le long des axes hydrauliques est déjà une manifestation du Tertiaire supérieur à travers l'affleurement de la cuirasse ferrugineuse du Pliocène, devenue gravillonnaire au fil des évolutions diagénétiques. Sa mise en place remonterait entre 5,3 et 1,8 millions d'années est le résultat selon Maignien (1958) et Millot (1964) d'une migration latérale du fer lessivé à l'amont et précipité à l'aval, dans des conditions oxydantes provoquant ainsi sa concentration dans la partie supérieure des profils latéritiques. Il a ensuite subi selon Michel (1973), un transport avant d'être cimentés sur le lieu d'épandage. Le tout se déroule dans un milieu tropical humide, mais à saisons contrastées dans le long terme. Ceci est attesté par les travaux de Tardy et Roquln (1990), qui a montré que les cuirasses des haut et moyen glacis sont respectivement attribuées au Miocène et au Pliocène (étant entendu que les cuirasses d'un même niveau présentent généralement un diachronisme). Par ailleurs, des sondages de prospection avaient signalé la présence de phosphates dans différentes parties de la moyenne vallée du Sénégal (F. Jacquet, 1936 ; Elouard, P., 1962, in BRGM, 1965 et 1984). A hauteur de Kanel (23,5 km de Ourossogui), les travaux de Baud (1936-1938) confirment que ces calcaires phosphatés plongent entre 5 et $11 \mathrm{~m}$ de profondeur : ce qui correspond à la profondeur d'affleurement de la cuirasse ferrugineuse du Pliocène sur certains endroits du ravin du Sud-est du bassin versant de Ourossogui. Ces sédiments d'origine marine signalés par la figure 11 correspondent donc à des apparitions de faciès de calcaires phosphatées dans cette partie du bassin versant.

Ce phénomène est amplifié par la dynamique structurale du bassin versant, sous tendue par des roches tendres, donc peu résistantes face aux pressions du fluide hydrique. A cause de leur texture poreuse (sables ogoliens en surface), les eaux de ravinement s'exercent sur des terrains légèrement inclinés et creusent des ravins et ravines qui acheminent les eaux dans les zones topographiquement faibles. La présence de graviers dans certains endroits du bassin atteste l'importance de la morphodynamique hydrique par ravinement, car le déplacement de ces individus granulométriques nécessite une force mécanique considérable. Les débits solides en transit fonctionnement par ailleurs comme abrasifs en modes de transport par roulage et par saltation (Sow, 2018). Au fur et à mesure que les ravinements creusent les axes hydrauliques, se démantèlent les faciès en doublure, laissant ainsi apparaitre des individus lithologiques en profondeur et témoins d'anciennes fluctuations morphoclimatiques, comme ces rares populations d'origine marine (calcaires phosphatés. Les ravins situés au piémont des collines de cuirasses du bassin versant de Ourossogui apparaissent comme d'excellents 
archives sédimentaires, permettant d'avoir des informations sur la paléolithologie des faciès en superposition, soit l'œil nu à travers les couples de résistance qui affleurent, soit consécutivement à des analyses granulométriques au laboratoire.

\section{Conclusion}

L’objectif de ce travail était de faire une caractérisation sédimentaire par granulométrie, dans l'optique de rétablir le contexte paléoenvironnementale des sédiments du bassin versant de Ourossogui. Le protocole méthodologique est fondé sur un échantillonnage et des analyses granulométriques au laboratoire, suivi d'un traitement statistique des résultats. Ces derniers aboutissent dans l'ensemble à une prédominance de la fraction sableuse. Sur la colonne de tamis utilisée, ces fractions concernent les particules dont le diamètre se situe entre $2 \mathrm{~mm}$ et $0,1 \mathrm{~mm}$ : sables très gros, sables gros, sables moyens, sables fins, sables très fins. Les sédiments sont issus du domaine continental excepté quelques-uns ayant une genèse marine. Dans l'ensemble, ces sédiments sont transportés par les eaux courantes (de ravinement et de ruissellement). Cette situation paléo-environnementale du bassin versant est à l'origine de sa forte dynamique structurale, marquée par une réactivation de la morphodynamique hydrique.

\section{References:}

1. Balzerek, H. (2003). Man-made flood disaster in the savanna town of Gombe,NE Nigeria; the natural hazard of gully erosion caused by urbanization dynamics and their periurban footprints. Erdkunde, (2), 94-109p.

2. Baud, L. (1936-1938). Rapports sur les phosphates de la vallée du fleuve Sénégal. Mission d'études du fleuve Sénégal. [Rapp. Inéd. Arch Dir. Mines AOF Dakar]

3. Bouzou, M. I., Maiga O., Ambouta, J. M., Sarr, B., Descroix, L., \& Adamou, M. A., (2009). Les conséquences géomorphologiques de l'occupation du sol et des changements climatiques dans un bassinversant rural sahélien. Sécheresse, (1), 145-152p.

4. BRGM (1967). Etude géochimique des eaux souterraines de l'Afrique de l'Ouest, Rapport BRGM-DAK 67-A12 fasc, 320p.

5. BRGM (1984). Plan minéral du Sénégal, Dakar (Ed.), 3 vol., 612p.

6. Chudeau, R. (1910). Notes sur la géologie du Soudan. Bull. Soc. Géol. Fr. Paris, (4). 16p.

7. Cosandey, C., \& Robinson, M. (2000). Hydrologie continentale, Paris, Armand Collin, 360 p. 
8. Dubois, J. (1967). Quelques observations utilisables pour la chronologie du Quaternaire au Sénégal et en Mauritanie, Congrès Panafricain de Préhistoire, Dakar

9. Elouard, P. (1962). Etude géologique et hydrogéologique des formations du sédimentaire Guebla mauritanien et de la vallée du Sénégal (Thèse Paris - Mémoires). BRGM. N07, Paris

10. Folk, R. L. \& Ward, W. C. (1957). Brazos rivers bar:a study in the significance of grain size parameters.Journ.Sc.Petrol,Vol.27, $\mathrm{N}^{\circ} 13-26$

11. Friedman G. M. (1962). Comparison of moment measures for sieving and thin-section data for sedimentary petrological studies. Jour. Sedim. Petrol, Vol. 32, 15 - 25 p.

12. Giresse, P. (2000). Successions of sea-level changes during the Pleistocene in Mauritania and Senegal distinguished by sedimentary fancies study and U/Th dating. Marine Geology, Vol. 2, 123-139 p.

13. Jacquet, F. (1936). L'Eocène et les phosphates dans la vallée du fleuve Sénégal. Séanc Acad Sci. ํ23, 272-274p.

14. Maignien, R. (1958). Le cuirassement des sols en Guinée, Afrique occidentale (Thèse Sciences Strasbourg). Illém. Seru. Carte géol. Als.Lorr., Strasbourg, no 16, 239 p., 24 fig., 3

15. Michel, P. (1973). Les bassins des fleuves Sénégal et de la Gambie (étude géomorphologique). Thèse d'Etat Mémoire IRD, Numéro 63, $752 \mathrm{p}$.

16. Millot, G. (1964). Géologie des argiles. Altérations-sédimentologiegéochimie. Paris, Masson, $499 \mathrm{p}$.

17. Moiola, R. J. \& Weiser, D. (1968). Textural parameters : an evaluation. Jour. Sedim. Petrol., Vol. 38, 45 - 53 p.

18. Sakho I. (2011), Evolution et fonctionnement hydro-sédimentaire de la lagune de la Somone, Petite Cote, Sénégal. Thèse de doctorat de géologie, Université de Rouen (France), 252 p.

19. Sow S. A. (2018), Morphodynamique hydrique par ravinement et ses conséquences dans le bassin versant de Ourossogui, Nord du Sénégal. Thèse de doctorat de Géographie, UGB, Sénégal, 328p.

20. Tardy, Y. \& Roquln C. (1990). Geochemistry and evolution of lateritic landscapes in Chesworth W. and Martini 1. P. Edit., Elsevier (sous presse).

21. Tricart J. (1956), Aspects géomorphologiques du delta du Sénégal. Revue de Géomorphologie Dynamique, N5, 65-85 pp.

22. Tossou E. M. (2019), Analyse Granulométrique et Cartographie des Sédiments Superficiels de la Lagune Côtière du Benin (Afrique de l'Ouest), European Scientific Journal, Vol 15, $\mathrm{N}^{\circ} 24$, Doi : 10.19044/esj.2019.v15n24p294 